\title{
Portuguese Hospital Anxiety and Depression Scale (HADS): Usefulness in Focal Epilepsy
}

\author{
R Meneses, J Pais-Ribeiro, A Martins da Silva, A Giovagnoli
}

\section{Citation}

R Meneses, J Pais-Ribeiro, A Martins da Silva, A Giovagnoli. Portuguese Hospital Anxiety and Depression Scale (HADS): Usefulness in Focal Epilepsy. The Internet Journal of Mental Health. 2007 Volume 5 Number 2.

\begin{abstract}
Objective: To explore the usefulness of the Portuguese HADS for outpatients with focal epilepsy. Methods: Ninety-nine outpatients were assessed using a socio-demographic and clinical questionnaire, the MHI-5, and the HADS (individual interview). Results: HADS proved to be understandable and well accepted by patients, with alpha ranges from 0.72 to 0.82 . It also correlated with $\mathrm{MHI}-5$ and some demographic and clinical variables.Conclusions: The results support the use of the Portuguese HADS as a screening tool for these patients (facial validity; internal consistency; construct validity), though the associations between HADS scores and some demographic and clinical variables question its validity. The results underscore the importance of assessing patient affective status, as anxiety and depression are common and provide additional insight into the association of anxiety/depression with certain demographic and clinical variables. The reasons for the inconsistencies between these results and published literature should be explored in future studies.
\end{abstract}

The work was done at Neurophysiology Department Hospital Geral de Santo António, Porto, Portugal.

Financial support from the Science and Technology Foundation (Portugal)/Fundação para a Ciência e a Tecnologia to the $\mathrm{PhD}$ research project in the context of which the data presented in this article was obtained (Grant PRAXIS XXI / BD / 18536 / 98 - Sub-Programa Ciência e Tecnologia do $2^{\circ}$ Quadro Comunitário de Apoio).

\section{INTRODUCTION}

\section{ANXIETY AND DEPRESSION IN (FOCAL) EPILEPSY}

Interictal anxiety, can affect as high as $66 \%$ of those with epilepsy, can be a reaction to the possibility of having a seizure or to the interferences of epilepsy on daily life $\left(_{1}, 2\right.$ ). Moreover, psychiatric and neuropsychological factors can interact, making patients daily functioning even more difficult $\left({ }_{3}\right)$. Since affective state can influence performance in tasks used to assess other functional potentialities and limitations, affective assessment is essential $\left({ }_{4},{ }_{5}\right)$ and can help optimize care. In fact, in Portugal, patients with epilepsy reported a need for specialized support to cope with several psychosocial issues, namely related to specific emotional worries ${ }_{6}{ }_{6}$.

Psychiatric symptoms are common in epilepsy patients ${ }_{7},{ }_{8}$
). Even though the majority of those with well controlled epilepsy do not show significant evidence of psychopathology, some (especially those with chronic epilepsy) present serious psychiatric disorders ( $\left.{ }_{9}\right)$. The additional burden of having a co-morbid mental disorder seems to be significant for individuals with chronic epilepsy ( 9 ). Furthermore, psychiatric symptoms can develop in relation to the seizures, but they are usually present as an interictal chronic condition $\left(_{2}\right)$. As depression and anxiety seem to be among the most common co-morbidities of epilepsy $\left({ }_{9},{ }_{10},{ }_{11},{ }_{12},{ }_{13},{ }_{14}\right)$, it is also crucial to consider them in epilepsy research, since any of these disorders may bias other clinical indicators and/or research variables: e.g., several perceptions and performances ( $\mathrm{cf}_{15}$ ).

Unfortunately, psychopathological symptoms are not regularly diagnosed, nor treated, in epilepsy patients, perhaps because health care professionals do not have enough time to do so $\left({ }_{9},{ }_{16},{ }_{17},{ }_{18},{ }_{19},{ }_{20}\right)$. For example, O'Donoghue et al. ${ }_{21}$ ) found that among individuals with one or more epileptic seizures, only a third of those classified as "definite or possible cases" according to the Hospital Anxiety and Depression Scale (HADS) had records of psychological symptoms in their clinical records of the previous two years. Thus, treatment and prevention of comorbid psychiatric disorders require instruments that do not disrupt the clinical routine. The HADS seems a very good 
candidate for such a role as far as anxiety and depression are concerned, with promising results reported in the literature.

The HADS showed no differences in anxiety between individuals with and without (partial) epilepsy $\left({ }_{22},{ }_{23}\right)$. On the other hand, in patients with other somatic diseases, including epilepsy, and post-natal women, the prevalence of anxiety symptoms, according to the HADS, was $11-22 \%$ ( $_{20}$ ). The same scale indicated that the prevalence of anxiety symptoms in patients with partial epilepsy was: none $=52 \%$, mild $=25 \%$, moderate $=16 \%$, and severe $=7 \%\left({ }_{24}\right)$.

The HADS also revealed signs of anxiety in over $40 \%$ of individuals with recently diagnosed epilepsy, without serious psychological or medical diseases ${ }_{25}$ ). In these individuals, the HADS also indicated that being younger, being a woman, and having more recent seizures is significantly associated with higher scores of anxiety, but not depression ( $\left.{ }_{25}\right)$. The anxiety score from the HADS correlated with seizure frequency, epilepsy duration, and sex in individuals taking antiepileptic medication $\left({ }_{26}\right)$. Sex was also an important factor explaining the variability of anxiety women were more likely to be anxious than men $\left.{ }_{26}\right)$.

In outpatients with newly diagnosed and chronic epilepsy, only the type of epilepsy was significantly associated with the State-Trait Anxiety Inventory-State index, whereas high seizure frequency, polypharmacy, symptomatic focal epilepsy, and female gender were positively correlated with the State-Trait Anxiety Inventory-Trait index ( 27 .

In adults with epilepsy in the community, the prevalence of anxiety (HADS) was 20.5\% (95\% CI: 16.9-24.1\%). It was associated with a current history of depression, perceived side effects of antiepileptic medication, lower educational attainment, chronic ill health, female gender, and unemployment. It was not associated with the duration of epilepsy $\left.{ }_{28}\right)$.

With regard to depression, the HADS showed no differences between individuals with and without (partial) epilepsy ${ }_{22}$, ${ }_{23}$ ). According to the same scale, the prevalence of depressive symptoms in patients with somatic diseases, namely epilepsy, and post-natal women was 11-19\% ( $\left.{ }_{20}\right)$. In a sample of patients with partial epilepsy, the HADS indicated a prevalence of: none $=62 \%$, mild $=20 \%$, moderate $=14 \%$, and severe $=4 \%\left({ }_{24}\right)$.

The HADS indicated signs of depression in more than $15 \%$ of subjects with recently diagnosed epilepsy $\left({ }_{25}\right)$. It also revealed that in individuals taking antiepileptic medication, depression correlated with seizure frequency, epilepsy duration, age at seizure onset, and age ${ }_{26}$ ).

The prevalence of depression (HADS) in a nonspecialist care-identified epilepsy population was $11.2 \%$ (95\% CI: $8.3-13.7 \%)$, i.e., greater than in the general population $\left(_{29}\right)$. Depression was most strongly associated with unemployment, but also with having had a recent seizure and perceived medication side effects ${ }_{29}$ ). It was not associated with gender, marital status, monotherapy or polytherapy ${ }_{29}$ ). In epilepsy outpatients, high seizure frequency, type of epilepsy syndrome and a high number of antiepileptic drugs (AEDs) were significantly and positively associated with depression (Beck Depression Inventory-21), while female gender showed a trend towards being significantly associated with depression $\left({ }_{27}\right)$.

The HADS indicated that in patients with somatic diseases, including epilepsy, and post-natal women, about half had comorbid depressive and anxiety symptoms $\left.{ }_{20}\right)$. In patients with temporal lobe epilepsy, depression and anxiety (Beck Depression Inventory and Self-Rating Anxiety Scale) were related to type of intervention (epilepsy surgery + AEDs or AEDs alone), underlying epileptogenic lesion, change of seizure control and AED ( $\left.{ }_{30}\right)$. The authors concluded that depression improved not because of surgery, but because of improved seizure control $\left({ }_{30}\right)$.

Maybe methodological differences explain the inconsistency of the complex relationship verified between epilepsy variables (e.g., seizure frequency and type, epileptogenic focus laterality) and depression $\left({ }_{14}\right)$. Nonetheless, there seem to be higher depression levels in individuals with temporal lobe epilepsy or psychomotor vs. generalized seizures $\left({ }_{14}\right)$.

In summary, research shows that anxiety and depression are associated with and predict important variables, including epilepsy patients' quality of life $\left({ }_{24},{ }_{31}, 3_{32},{ }_{33},{ }_{34},{ }_{35}\right)$. Therefore, psychological treatment of negative affect may improve patients' quality of life $\left.{ }_{36}\right)$.

\section{ANXIETY, DEPRESSION, AND ITS ASSESSMENT: THE HADS}

The detailed psychometric properties of the instrument(s) chosen for a particular study or intervention focusing on anxiety and/or depression are rarely adequately reported, which is surprising when the literature shows a wide variation in terms of scale construction and composition ${ }_{37}$ 
) .

The HADS has some remarkable characteristics: a) it has been translated to a number of languages, and is widely used, in several cultures, with numerous populations, of various age groups, and purposes (cross-sectional and longitudinal studies); b) it is short and easy/quick to administer (2-6 minutes) and score; c) it is a self-report scale; d) it separates anxiety from depression; e) it emphasises anhedonia; f) it avoids symptoms likely to appear in somatic diseases (e.g., weight loss); g) it is easily understandable and accepted by patients, since it does not cover serious psychopathologic symptoms (also avoids floor effects); h) it has potential as a screening tool, providing scores suggestive of the probability of anxiety/depression, as well as its levels; i) it provides ranges suggestive of the disorder severity; $\mathrm{j}$ ) it provides a record of longitudinal data; k) it has satisfactory psychometric properties, namely face validity; and 1) since the scores are influenced by the intervention, it can give some therapeutic guidance $\left({ }_{38},{ }_{39},{ }_{40}\right.$ $\left.,_{41},{ }_{42},{ }_{43},{ }_{44},{ }_{45}\right)$. It is able to predict mood over a year or more, compliance, quality of life, 1-year mortality (HADSDepression) and physical symptoms (HADS-Anxiety) ( 40,46 ) .

The HADS was developed based on data gathered from individuals aged 16 to 65 years, men and women, with a variety of physical pathologies/complaints, and from hospital staff $\left({ }_{47}\right)$. The HADS, developed for nonpsychiatric hospital use, adequately reached its goal: to assess psychological states with a minimum of disturbance due to somatic disease symptoms $\left({ }_{41},{ }_{45},{ }_{47}\right)$. Its subsequent use showed that it is a useful and reliable measure to screen for clinically significant anxiety and depression; and that it is a valid measure of the severity of these conditions $\left({ }_{45},{ }_{47}\right)$. It can also be used with adolescents between 12 and 17 years of age $\left({ }_{48}\right)$.

Consequently, this primarily clinical tool is also widely used in clinical research $\left({ }_{37},{ }_{44},{ }_{49}\right)$. It is worth stressing that the HADS does not aim at providing psychiatric diagnosis, but to emphasise the separation between anxious and depressive states $\left({ }_{44}\right)$. In clinical settings, high scores should be followed by an in-depth psychiatric interview ${ }_{40}$ ).

The HADS focuses on the generalized anxiety state and not necessarily on the anxiety for a specific situation (e.g, hospital visit); it covers the anxious state, restlessness and anxious thoughts $\left({ }_{44},{ }_{45}\right)$. The HADS-Anxiety is mainly constituted by mood, followed by behaviour and, finally, cognition, ignoring the arousal and somatic aspects; the HADS-Depression focus on the loss of pleasure, i.e., mainly in the loss of interest and diminished pleasure response (anhedonia - 85.7\% - and fatigue - 14.3\%) $\left({ }_{37},{ }_{42},{ }_{44},{ }_{45},{ }_{47}\right.$, $49)$.

The HADS refers to the previous week $\left({ }_{45},{ }_{47}\right)$. It comprises 14 multiple-choice items, equally divided by two subscales (Anxiety and Depression), with independent scoring ( $\left.{ }_{45}\right)$. Unlike typical state instruments, it is sufficiently stable to resist to situational influences, but it is less stable than typical trace scales, in a broader time period $\left({ }_{40}\right)$.

Even though the HADS authors and some research data do not recommend the use of a global score $\left({ }_{40},{ }_{44},{ }_{45},{ }_{47}\right)$, there is also data supporting the use of three scores (global, anxiety, and depression) $\left({ }_{15},{ }_{41}\right)$. Moreover, research shows that the HADS allows the separate assessment of anxiety and depression, but there is some overlap, mainly due to an actual overlap of symptoms $\left({ }_{40}\right)$. In fact, some data support the existence of common factors/dimensions shared by anxiety and depression $\left({ }_{50}\right)$.

As each item is answered on a 4-point scale $(0-3)$, possible scores range from 0 to 21 (anxiety/depression) and 0-42 (negative affect $\left({ }_{15}\right)$ ). In the absence of consensus $\left({ }_{38},{ }_{51},{ }_{52}\right.$ ), scores are usually interpreted as: 0-7 normal, 8-10 low, 11-14 moderate, and 15-21 severe for anxiety or depression ( $\left.{ }_{43},{ }_{45}\right)$. The validation process of the Portuguese HADS version shows psychometric properties similar to those in international studies ${ }_{53}$ ).

Research and clinical practice underscore that there is still much to be done and to study to adequately assess, understand, prevent, and treat psychiatric co-morbidity in epilepsy $\left({ }_{9},{ }_{50}\right)$. Routine affective assessment and the consideration of its relevance for the general presentation should be included in every clinical assessment $\left({ }_{54}\right)$. Being so, the aim of the present study was to explore the usefulness of the HADS-Portuguese version as a screening instrument and, consequently, intervention aid for Portuguese outpatients with focal epilepsy.

\section{METHOD}

A consecutive sample of 99 outpatients with clinical evidence of focal epilepsy, from the Epilepsy Unit of Hospital Geral de Santo António (Porto, Portugal), was assessed. More than half were women, married/cohabiting, and professionally active (cf. Table 1), even though there was a considerable variability in terms of profession. The 
sample also varied greatly in terms of age (14-70 yrs) and schooling (0-17 yrs; cf. Table 1).

\section{Figure 1}

Table 1: Socio-demographic Characteristics of the Participants (=99)

\begin{tabular}{|l|c|c|c|c|}
\hline & Frequency & Percentage & $\underline{\text { M }}$ & $\underline{\text { SD }}$ \\
\hline Sex & & & & \\
\hline Females & 55 & 55.6 & & \\
\hline Males & 44 & 44.4 & & \\
\hline Marital Status & & & & \\
\hline Married/cohabiting & 62 & 62.6 & & \\
\hline Single & 32 & 32.3 & & \\
\hline Divorced/Separated & 3 & 3.0 & & \\
\hline Widow & 2 & 2.0 & & \\
\hline Professional Status & & & & \\
\hline Active & 67 & 67.6 & & \\
\hline Student & 15 & 15.2 & & \\
\hline Retired & 13 & 13.1 & & \\
\hline Unemployed & 4 & 4.0 & & 36.41 \\
\hline Age & & & 7.62 & \\
\hline Schooling & & & & \\
\hline
\end{tabular}

Note: $\mathrm{M}=$ mean, $\mathrm{SD}=$ standard deviation
In clinical terms, the majority of participants: were on monotherapy with AEDs, had normal AED serum levels, reported no side-effects, had done an electroencephalogram (EEG) and a CT (computerized tomography) scan previously, had done no MRI (magnetic resonance imaging), had no lesional epileptic focus, had the last seizure more than a month before assessment, had a single seizure type, and had one or less than one seizure a month (cf. Table 2). The sample encompasses individuals with very diverse disease onset $\left(\mathrm{M}_{(97)}=15.59, \mathrm{SD}=10.91,0.17-46.00 \mathrm{yrs}\right)$ and age at disease onset $\left(M_{(97)}=20.70, S D=13.39,0-57 \mathrm{yrs}\right)$.

The most frequently used AEDs were: phenytoin, valproic acid, carbamazepine, clobazam, and primidone. 


\section{Figure 2}

Table 2: Clinical Characteristics of the Participants (=99)

\begin{tabular}{|c|c|c|}
\hline & Frequency & Percentage \\
\hline \multicolumn{3}{|l|}{ AED } \\
\hline Without & 8 & 8.1 \\
\hline Monotherapy* & 53 & 53.5 \\
\hline Bitherapy & 28 & 28.3 \\
\hline Politherapy & 10 & 10.1 \\
\hline \multicolumn{3}{|l|}{ AED serum levels } \\
\hline Subtherapeutic & 17 & 17.2 \\
\hline Normal & 50 & 50.5 \\
\hline Toxic & 5 & 5.1 \\
\hline Missing & 27 & 27.3 \\
\hline \multicolumn{3}{|l|}{ Side-effects } \\
\hline Without & 75 & 75.8 \\
\hline With & 16 & 16.2 \\
\hline Missing & 8 & 8.1 \\
\hline \multicolumn{3}{|l|}{ 1st EEG } \\
\hline No & 86 & 86.9 \\
\hline Yes & 8 & 8.1 \\
\hline Missing & 5 & 5.1 \\
\hline \multicolumn{3}{|l|}{ CT scan } \\
\hline Without & 18 & 18.2 \\
\hline With & 81 & 81.8 \\
\hline \multicolumn{3}{|l|}{ MRI } \\
\hline Without & 51 & 51.5 \\
\hline With & 48 & 48.5 \\
\hline \multicolumn{3}{|c|}{ Lesional epileptic focus } \\
\hline Yes & 35 & 35.4 \\
\hline No & 49 & 49.5 \\
\hline Missing & 15 & 15.2 \\
\hline \multicolumn{3}{|l|}{ Last seizure** } \\
\hline That day & 1 & 1.0 \\
\hline$<1$ week & 17 & 17.2 \\
\hline$<1$ month & 22 & 22.2 \\
\hline$<1$ year & 28 & 28.3 \\
\hline$\geq 1$ year & 28 & 28.3 \\
\hline Missing & 3 & 3.0 \\
\hline \multicolumn{3}{|l|}{ Single seizure type } \\
\hline No & 29 & 29.3 \\
\hline Yes & 67 & 67.7 \\
\hline Missing & 3 & 3.0 \\
\hline \multicolumn{3}{|c|}{ Seizure frequency*** } \\
\hline Daily & 4 & 4.0 \\
\hline$>1 /$ week & 6 & 6.1 \\
\hline$\leq 1 /$ week & 2 & 2.0 \\
\hline$>1 /$ month & 17 & 17.2 \\
\hline$\leq 1 /$ month & 11 & 11.1 \\
\hline$>1 /$ year & 8 & 8.1 \\
\hline$\leq 1 /$ year & 23 & 23.2 \\
\hline Without seizures & 16 & 16.2 \\
\hline Missing & 12 & 12.1 \\
\hline
\end{tabular}

Note: AED - antiepileptic drug; EEG - electroencephalogram; CT computerized tomography; MRI - magnetic resonance imaging

* Monotherapy - use of only one AED; Bitherapy - use of two AEDs: Polytherapy - use of more than two AEDs

** "Last seizure" categories are completely separate categories;

*** "Seizure frequency" categories are completely separate categories 
A socio-demographic and clinical questionnaire, the Mental Health Questionnaire-5 (MHI-5), and the HADS were administered in the context of an individual interview, due to the expected low literacy of some participants. Some clinical data was obtained from the patients' physicians, through the consultation of their medical records. Internationally accepted ethical standards were followed, namely adherence to the ethical principles of the Declaration of Helsinki (for a detailed report of the procedure, see Meneses $\left({ }_{15}\right)$ ).

The Portuguese version of the MHI-5 has a structure similar to the original version, making it possible to use it in a similar way $\left({ }_{55}\right)$. It has five items representing four dimensions of mental health (Anxiety, Depression, Lost of Control Emotional-Behavioural, and Psychological Wellbeing), so three items are from the Distress Scale and two from the Positive Well-being Scale of the MHI; it can be used independently, as a screening instrument, or included in other scales (e.g., SF-36); research has shown its utility as a screening instrument $\left({ }_{55}\right)$. It is scored with higher scores indicating a better health state ${ }_{56}$ ).

It is worth stressing that the MHI-5 was chosen as a marker for the HADS construct validity because the MHI-5 is brief, is frequently used worldwide in health care settings, namely as part of the SF-36 ( $\left.{ }_{15}\right)$, and has been proved useful as a screening instrument. Nevertheless, unlike a psychiatric interview (especially one guided by a validated scale), the MHI-5 is not considered a gold standard instrument regarding the HADS capacity to identify symptoms of anxiety and/or depression. When using the MHI-5 as a validity marker regarding screening per se, one should remember that the MHI-5 and the HADS are built with different construct aspects (cf. what was previously said about both instruments).

\section{RESULTS}

Table 3 shows the minimum, maximum, mean, and standard-deviation values obtained by the participants in the HADS and MHI-5 (sub)scales and items. It also shows the internal consistency of each (sub)scale (Cronbach alpha). Consequently, it demonstrates there was considerable variability in the sample as far as these mental health indicators were concerned. It also indicates that the internal consistency of the (sub)scales can be considered from acceptable $(>0.70)$ to good $(>0.80)$.

\section{Figure 3}

Table 3: Minimum, Maximum, Mean, Standard-deviation, by Item and Scale, and Internal Consistency (Cronbach Alpha) of each Scale (=99)

\begin{tabular}{|l|c|c|c|c|c|}
\hline & Minimum & Maximum & $\underline{\underline{M}}$ & $\underline{\text { SD }}$ & $\underline{\text { alpha }}$ \\
\hline HADS-Anxiety & 1.00 & 19.00 & 8.60 & 4.17 & 0.72 \\
\hline HADS_1 & 0 & 3 & 1.31 & 0.88 & \\
\hline HADS_3 & 0 & 3 & 1.13 & 1.12 & \\
\hline HADS_5 & 0 & 3 & 1.63 & 1.03 & \\
\hline HADS_7 & 0 & 3 & 1.32 & 1.02 & \\
\hline HADS_9 & 0 & 3 & 0.80 & 0.78 & \\
\hline HADS_11 & 0 & 3 & 1.70 & 1.10 & \\
\hline HADS_13 & 0 & 3 & 0.71 & 0.87 & \\
\hline HADS- & 0.00 & 18.00 & 5.18 & 4.18 & 0.78 \\
\hline Depression & 0 & 3 & 0.52 & 0.89 & \\
\hline HADS_2 & 0 & 3 & 0.48 & 0.72 & \\
\hline HADS_4 & 0 & 3 & 0.73 & 0.81 & \\
\hline HADS_6 & 0 & 3 & 1.35 & 0.96 & \\
\hline HADS_8 & 0 & 3 & 0.56 & 0.87 & \\
\hline HADS_10 & 0 & 3 & 0.74 & 1.02 & \\
\hline HADS_12 & 0 & 3 & 0.81 & 1.08 & \\
\hline HADS_14 & 1.0 & 34.00 & 13.78 & 7.30 & 0.82 \\
\hline HADS-Total & 2.00 & 6.0 & 4.36 & 1.50 & \\
\hline MHI-5 & 6.00 & 30.00 & 19.67 & 6.15 & 0.82 \\
\hline MHI-5_1 & 1.0 & 6.0 & 3.67 & 1.628 & \\
\hline MHI-5_2 & 1.0 & 6.0 & 4.58 & 1.52 & \\
\hline MHI-5_3 & 1.0 & 6.0 & 3.43 & 1.64 & \\
\hline MHI-5_4 & 1.0 & 3.63 & 1.74 & \\
\hline MHI-5_5 & 1.0 & 6.0 & & & \\
\hline & & & & \\
\hline
\end{tabular}

Table 4 illustrates a more important clinical indicator of the sample, considering the most recent cut-off values of the English language version of the HADS $\left({ }_{43},{ }_{45}\right)$. It shows a significant number of cases of anxiety or depression, mostly anxiety, according to the HADS.

\section{Figure 4}

Table 4: Frequencies and Percentages of Participants in the Different Severity Classes of the HADS (=99)

\begin{tabular}{|l|c|c|c|c|c|c|c|c|}
\hline & \multicolumn{2}{|c|}{ Nomal (0-7) } & \multicolumn{2}{c|}{ Low (8-10) } & \multicolumn{2}{c|}{ Moderate (11-14) } & \multicolumn{2}{c|}{ Severe (15-21) } \\
\cline { 2 - 9 } & $\underline{\mathbf{N}}$ & $\%$ & $\underline{\mathrm{N}}$ & $\%$ & $\underline{\mathrm{N}}$ & $\%$ & $\underline{\mathrm{N}}$ & $\%$ \\
\hline HADS-Anxiety & 42 & 42.4 & 25 & 25.3 & 23 & 23.2 & 9 & 9.1 \\
\hline HADS-Depression & 75 & 75.8 & 13 & 13.1 & 6 & 6.1 & 5 & 5.0 \\
\hline
\end{tabular}

Table 5a and 5b show the correlations between HADS and MHI-5 items and scores obtained by the participants. Of all the possible correlations, only 11 were not statistically significant: 10 between HADS and MHI-5 items and one between item 11 of the HADS and HADS-Depression. 


\section{Figure 5}

Table 5a: Correlations (2-tailed) Between HADS and MHI-5 Items and Scores (=99)

\begin{tabular}{|l|c|c|c|c|c|c|c|c|c|c|}
\hline & \multicolumn{2}{|c|}{ MHI-5_1 } & \multicolumn{2}{c|}{ MHI-5_2 } & \multicolumn{2}{c|}{ MHI-5_3 } & \multicolumn{2}{c|}{ MHI-5_4 } & \multicolumn{2}{|c|}{ MHI-5_5 } \\
\hline & $\mathrm{I}$ & $\mathrm{R}$ & $\mathrm{I}$ & $\mathrm{R}$ & $\mathrm{I}$ & $\mathrm{R}$ & $\mathrm{I}$ & $\mathrm{R}$ & $\mathrm{I}$ & $\mathrm{R}$ \\
\hline HADS_1 & -0.59 & $<0.001$ & -0.31 & 0.002 & -0.61 & $<0.001$ & -0.51 & $<0.001$ & -0.46 & $<0.001$ \\
\hline HADS_2 & -0.14 & 0.184 & -0.44 & $<0.001$ & -0.19 & 0.058 & -0.36 & $<0.001$ & -0.43 & $<0.001$ \\
\hline HADS_3 & -0.34 & $<0.001$ & -0.37 & $<0.001$ & -0.20 & 0.043 & -0.46 & $<0.001$ & -0.38 & $<0.001$ \\
\hline HADS_4 & -0.09 & 0.391 & -0.51 & $<0.001$ & -0.25 & 0.013 & -0.34 & 0.001 & -0.39 & $<0.001$ \\
\hline HADS_5 & -0.44 & $<0.001$ & -0.42 & $<0.001$ & -0.38 & $<0.001$ & -0.39 & $<0.001$ & -0.48 & $<0.001$ \\
\hline HADS_6 & -0.24 & 0.030 & -0.55 & $<0.001$ & -0.30 & 0.003 & -0.51 & $<0.001$ & -0.61 & $<0.001$ \\
\hline HADS_7 & -0.33 & 0.001 & -0.35 & $<0.001$ & -0.40 & $<0.001$ & -0.39 & $<0.001$ & -0.50 & $<0.001$ \\
\hline HADS_8 & -0.17 & 0.090 & -0.24 & 0.017 & -0.24 & 0.019 & -0.30 & 0.003 & -0.27 & 0.007 \\
\hline HADS_9 & -0.26 & 0.009 & -0.33 & 0.001 & -0.25 & 0.013 & -0.35 & $<0.001$ & -0.20 & 0.048 \\
\hline HADS_10 & -0.23 & 0.023 & -0.28 & 0.005 & -0.41 & $<0.001$ & -0.32 & 0.001 & -0.19 & 0.057 \\
\hline HADS_11 & -0.16 & 0.115 & -0.16 & 0.107 & 0.03 & 0.737 & -0.24 & 0.016 & -0.15 & 0.137 \\
\hline HADS_12 & -0.22 & 0.029 & -0.29 & 0.003 & -0.24 & 0.018 & -0.22 & 0.030 & -0.38 & $<0.001$ \\
\hline HADS_13 & -0.24 & 0.019 & -0.36 & $<0.001$ & -0.22 & 0.031 & -0.39 & $<0.001$ & -0.25 & 0.013 \\
\hline HADS_14 & -0.24 & 0.016 & -0.39 & $<0.001$ & -0.19 & 0.060 & -0.25 & 0.011 & -0.38 & $<0.001$ \\
\hline HADS- & -0.55 & $<0.001$ & -0.54 & $<0.001$ & -0.46 & $<0.001$ & -0.63 & $<0.001$ & -0.57 & $<0.001$ \\
\hline Anxiety & & & & & & & & & & \\
\hline HADS- & -0.29 & 0.004 & -0.58 & $<0.001$ & -0.39 & $<0.001$ & -0.48 & $<0.001$ & -0.57 & $<0.001$ \\
Depression & & & & & & & & & & \\
\hline HADS-Total & -0.48 & $<0.001$ & -0.64 & $<0.001$ & -0.48 & $<0.001$ & -0.64 & $<0.001$ & -0.65 & $<0.001$ \\
\hline MHI-5 & 0.74 & $<0.001$ & 0.79 & $<0.001$ & 0.70 & $<0.001$ & 0.83 & $<0.001$ & 0.79 & $<0.001$ \\
\hline & & & & & & & & & & \\
\hline
\end{tabular}

\section{Figure 6}

Table 5b: Correlations (2-tailed) Between HADS and MHI-5 Items and Scores (=99) (Continued)

\begin{tabular}{|c|c|c|c|c|c|c|c|c|}
\hline & \multicolumn{2}{|c|}{$\begin{array}{l}\text { HADS- } \\
\text { Anxiety }\end{array}$} & \multicolumn{2}{|c|}{$\begin{array}{c}\text { HADS- } \\
\text { Depression }\end{array}$} & \multicolumn{2}{|c|}{ HADS-Total } & \multicolumn{2}{|c|}{ MHI-5 } \\
\hline & $\underline{r}$ & p & $\underline{r}$ & p & $\underline{I}$ & p & I & p \\
\hline HADS_1 & 0.61 & $<0.001$ & 0.32 & 0.001 & 0.53 & $<0.001$ & -0.65 & $<0.001$ \\
\hline HADS_2 & 0.30 & 0.003 & 0.73 & $<0.001$ & 0.59 & $<0.001$ & -0.41 & $<0.001$ \\
\hline HADS_3 & 0.72 & $<0.001$ & 0.35 & $<0.001$ & 0.61 & $<0.001$ & -0.46 & $<0.001$ \\
\hline HADS_ 4 & 0.34 & 0.001 & 0.78 & $<0.001$ & 0.64 & $<0.001$ & -0.41 & $<0.001$ \\
\hline HADS_5 & 0.62 & $<0.001$ & 0.34 & 0.001 & 0.55 & $<0.001$ & -0.55 & $<0.001$ \\
\hline HADS_6 & 0.52 & $<0.001$ & 0.72 & $<0.001$ & 0.71 & $<0.001$ & -0.57 & $<0.001$ \\
\hline HADS_7 & 0.55 & $<0.001$ & 0.36 & $<0.001$ & 0.52 & $<0.001$ & -0.52 & $<0.001$ \\
\hline HADS_8 & 0.39 & $<0.001$ & 0.59 & $<0.001$ & 0.56 & $<0.001$ & -0.31 & 0.002 \\
\hline HADS_9 & 0.66 & $<0.001$ & 0.41 & $<0.001$ & 0.61 & $<0.001$ & -0.36 & $<0.001$ \\
\hline HADS_10 & 0.40 & $<0.001$ & 0.58 & $<0.001$ & 0.56 & $<0.001$ & -0.37 & $<0.001$ \\
\hline HADS_11 & 0.49 & $<0.001$ & 0.16 & 0.112 & 0.37 & $<0.001$ & -0.18 & 0.084 \\
\hline HADS_12 & 0.26 & 0.010 & 0.61 & $<0.001$ & 0.50 & $<0.001$ & -0.35 & $<0.001$ \\
\hline HADS_13 & 0.67 & $<0.001$ & 0.37 & $<0.001$ & 0.60 & $<0.001$ & $=0.37$ & $<0.001$ \\
\hline HADS_14 & 0.27 & 0.006 & 0.65 & $<0.001$ & 0.53 & $<0.001$ & -0.38 & $<0.001$ \\
\hline $\begin{array}{l}\text { HADS- } \\
\text { Anxiety }\end{array}$ & & & 0.53 & $<0.001$ & 0.87 & $<0.001$ & -0.71 & $<0.001$ \\
\hline $\begin{array}{l}\text { HADS- } \\
\text { Depression }\end{array}$ & 0.53 & $<0.001$ & & & 0.88 & $<0.001$ & -0.60 & $<0.001$ \\
\hline $\begin{array}{l}\text { HADS- } \\
\text { Total }\end{array}$ & 0.87 & $<0.001$ & 0.88 & $<0.001$ & & & & \\
\hline MHI-5 & -0.71 & $<0.001$ & -0.60 & $<0.001$ & -0.75 & $<0.001$ & & \\
\hline
\end{tabular}

With regard to the relationship between HADS and MHI-5 and the socio-demographic characteristics of the sample, there was no statistically significant correlation between: a) HADS-Anxiety and age, or schooling; b) HADS-Depression and schooling; c) HADS-Total and age, or schooling; and d) MHI-5 and schooling. HADS-Depression $(\mathrm{r}(99)=0.22$, $\mathrm{p}=0.03)$ and MHI-5 correlated with age $(\mathrm{r}(99)=-0.20$, $\mathrm{p}=0.04$ ). Only one item (item 12) of the HADS correlated with age $(\mathrm{r}(99)=0.20, \mathrm{p}<0.05)$; while two others correlated with schooling (item $11-\mathrm{r}(99)=-0.35, \mathrm{p}<0.001-$ and 14 $\mathrm{r}(99)=-0.25, \mathrm{p}<0.01)$.

There were statistically significant differences between men and women in some HADS items (1, 3, 7, 9, 10, and 13), HADS-Anxiety, HADS-Depression, HADS-Total, and MHI-5 (cf. Table 6). Women always had higher scores than men in these HADS indicators. For MHI-5, men reported better mental health.

\section{Figure 7}

Table 6: Differences between Men and Women in HADS Items and Scores and in MHI-5 (2-tailed)

\begin{tabular}{|c|c|c|c|c|c|c|c|}
\hline & \multicolumn{2}{|c|}{$\begin{array}{c}\text { Men } \\
(\mathrm{N}=44)\end{array}$} & \multicolumn{2}{|c|}{$\begin{array}{l}\text { Women } \\
(\mathrm{N}=55)\end{array}$} & \multirow[b]{2}{*}{$\underline{\mathrm{t}}$} & \multirow[b]{2}{*}{$\underline{\mathrm{df}}$} & \multirow[b]{2}{*}{$\mathrm{p}$} \\
\hline & $\underline{\mathrm{M}}$ & $\underline{\mathrm{SD}}$ & $\underline{\mathrm{M}}$ & $\underline{\mathrm{SD}}$ & & & \\
\hline HADS_1 & 1.02 & 0.76 & 1.55 & 0.90 & 3.130 & 97 & 0.002 \\
\hline HADS_2 & 0.39 & 0.87 & 0.62 & 0.89 & 1.300 & 97 & 0.197 \\
\hline HADS_3 & 0.68 & 0.88 & 1.49 & 1.17 & 3.922 & 97 & $<0.001$ \\
\hline HADS_4 & 0.36 & 0.65 & 0.58 & 0.76 & 1.509 & 97 & 0.135 \\
\hline HADS_5 & 1.45 & 1.09 & 1.76 & 0.96 & 1.499 & 97 & 0.137 \\
\hline HADS_6 & 0.73 & 0.90 & 0.73 & 0.73 & $<0.001$ & 97 & 1.000 \\
\hline HADS_7 & 0.89 & 0.97 & 1.67 & .92 & 4.116 & 97 & $<0.001$ \\
\hline HADS_8 & 1.18 & 0.97 & 1.49 & 0.94 & 1.602 & 97 & 0.112 \\
\hline HADS_9 & 0.57 & 0.59 & 0.98 & 0.87 & 2.697 & 97 & 0.008 \\
\hline HADS_10 & 0.36 & 0.75 & 0.71 & 0.94 & 2.038 & 97 & 0.044 \\
\hline HADS_11 & 1.68 & 1.18 & 1.71 & 1.05 & 0.122 & 97 & 0.903 \\
\hline HADS_12 & 0.61 & 0.97 & 0.84 & 1.05 & 1.085 & 97 & 0.281 \\
\hline HADS_13 & 0.50 & 0.73 & 0.87 & 0.94 & 2.152 & 97 & 0.034 \\
\hline HADS_14 & 0.61 & 1.02 & 0.96 & 1.11 & 1.622 & 97 & 0.108 \\
\hline HADS-Anxiety & 6.80 & 3.48 & 10.04 & 4.14 & 4.151 & 97 & $<0.001$ \\
\hline $\begin{array}{l}\text { HADS- } \\
\text { Depression }\end{array}$ & 4.25 & 3.98 & 5.93 & 4.22 & 2.016 & 97 & 0.047 \\
\hline HADS-Total & 11.05 & 6.49 & 15.96 & 7.22 & 3.521 & 97 & 0.001 \\
\hline MHI-5 & 21.86 & 5.29 & 17.91 & 6.28 & 3.338 & 97 & 0.001 \\
\hline
\end{tabular}

There were no statistically significant differences between married/cohabiting and single participants for HADSAnxiety, HADS-Total, or MHI-5. With regard to HADSDepression, the 62 married participants $(M=5.76)$ had a 
statistically significant higher mean than the 32 single ones $(\mathrm{M}=3.94 ; \mathrm{t}(86.230)=2.282, \mathrm{p}=0.03)$. As far as HADS items , married participants had a statistically higher mean for item $5(\mathrm{M}=1.73)$ than single participants $(\mathrm{M}=1.28, \mathrm{t}(92)=2.033$, $\mathrm{p}=0.045$ )

There were no statistically significant differences between those professionally active $(n=67)$ and those inactive $(n=32)$ for HADS-Anxiety, HADS-Depression, HADS-Total, and MHI-5. Item 14 differentiated professionally active from professionally inactive participants: active ones had a statistically higher mean ( $\mathrm{M}=0.99$ vs. $\mathrm{M}=0.44$, $\mathrm{t}(87.592)=2.804, \mathrm{p}=0.006)$.

With regard to clinical variables, there were no statistically significant correlations between any HADS item or score and disease onset. There was also no correlation between HADS-Anxiety and age at disease onset. MHI-5 did not correlate with disease onset or age at disease onset. HADSDepression $(\mathrm{r}(97)=0.21, \mathrm{p}=0.04)$, HADS-Total $(\mathrm{r}(97)=0.23$, $\mathrm{p}=0.02)$, item $1(\mathrm{r}(97)=0.20, \mathrm{p}<0.05)$, item $4(\mathrm{r}(97)=0.23$, $\mathrm{p}=0.02)$, and item $11(\mathrm{r}(97)=0.29, \mathrm{p}=0.005)$ correlated with age at disease onset.

There were no statistically significant differences between subjects on monotherapy $(\mathrm{n}=53)$ and taking more than one AED ( $n=38)$ with regard to HADS-Anxiety, HADSDepression, HADS-Total, or any HADS item. Those on monotherapy $(\mathrm{M}=20.74)$ had better mental health than those on polytherapy $(\mathrm{M}=18.08, \mathrm{t}(89)=2.094, \mathrm{p}=0.04)$.

There were no statistically significant differences between those who had the last seizure less than a month before assessment and those who had it more than a month before assessment in HADS-Anxiety. Those who had the last seizure less than a month before assessment had a statistically higher HADS-Depression mean, HADS-Total mean, and a statistically higher mean in items $4,5,6,7,8$, 12 , and 14 than those who had it more than a month before assessment. Those with a more recent seizure also had worse (lower) mental health according to the MHI-5 (cf. Table 7).

\section{Figure 8}

Table 7: Differences between Participants with a More or Less Recent Last Seizure in HADS Items and Scores and in MHI-5 (2-tailed)

\begin{tabular}{|c|c|c|c|c|c|c|c|}
\hline & \multicolumn{2}{|c|}{$\begin{array}{c}\text { More Recent } \\
\text { Seizure } \\
(\underline{N}=40)\end{array}$} & \multicolumn{2}{|c|}{$\begin{array}{c}\text { Less Recent } \\
\text { Seizure } \\
(\underline{N}=56)\end{array}$} & \multirow[b]{2}{*}{$\underline{t}$} & \multirow[b]{2}{*}{ df } & \multirow[b]{2}{*}{ p } \\
\hline & $\underline{\mathrm{M}}$ & $\underline{\mathrm{SD}}$ & $\underline{\mathrm{M}}$ & $\underline{\mathrm{SD}}$ & & & \\
\hline HADS-Anxiety & 9.33 & 4.06 & 8.00 & 4.13 & 1.561 & 94 & 0.122 \\
\hline $\begin{array}{l}\text { HADS- } \\
\text { Depression }\end{array}$ & 7.08 & 4.05 & 3.93 & 3.87 & 3.851 & 94 & $<0.001$ \\
\hline HADS-Total & 16.40 & 6.79 & 11.93 & 7.18 & 3.075 & 94 & 0.003 \\
\hline HADS_1 & 1.50 & 0.72 & 1.16 & 0.93 & 1.933 & 94 & 0.056 \\
\hline HADS_2 & 0.73 & 0.99 & 0.38 & 0.80 & 1.917 & 94 & 0.058 \\
\hline HADS_3 & 1.18 & 1.08 & 1.11 & 1.14 & 0.294 & 94 & 0.770 \\
\hline HADS_4 & 0.73 & 0.78 & 0.32 & 0.64 & 2.686 & 73 & 0.009 \\
\hline HADS_5 & 1.88 & 1.02 & 1.45 & 1.03 & 2.025 & 94 & 0.046 \\
\hline HADS_6 & 1.08 & 0.86 & 0.50 & 0.69 & 3.639 & 94 & $<0.001$ \\
\hline HADS_7 & 1.63 & 1.06 & 1.07 & 0.93 & 2.716 & 94 & 0.008 \\
\hline HADS_8 & 1.63 & 0.98 & 1.23 & 0.89 & 2.040 & 94 & 0.04 \\
\hline HADS_9 & 0.73 & 0.75 & 0.86 & 0.80 & 0.821 & 94 & 0.414 \\
\hline HADS_10 & 0.70 & 0.91 & 0.48 & 0.85 & 1.199 & 94 & 0.233 \\
\hline HADS_11 & 1.68 & 1.19 & 1.68 & 1.05 & 0.016 & 94 & 0.988 \\
\hline HADS_12 & 1.03 & 1.12 & 0.54 & 0.89 & 2.377 & 94 & 0.02 \\
\hline HADS_13 & 0.75 & 0.93 & 0.68 & 0.83 & 0.395 & 94 & 0.694 \\
\hline HADS_14 & 1.20 & 1.20 & 0.48 & 0.83 & 3.260 & 65 & 0.002 \\
\hline MHI-5 & 17.08 & 5.58 & 21.68 & 5.57 & -3.991 & 94 & $<0.001$ \\
\hline
\end{tabular}

There were no statistically significant differences between those who had one seizure type $(n=67)$ and those who had more than one seizure type $(n=29)$ in terms of HADSAnxiety, HADS-Depression, HADS-Total, or MHI-5. Nevertheless, they differed in items 3 and 5 of the HADS: those with more than one seizure type had a higher mean on item $3(\mathrm{M}=1.59$ vs. $\mathrm{M}=0.94, \mathrm{t}(94)=2.701, \mathrm{p}=0.008)$ and 5 $(\mathrm{M}=2.14$ vs. $\mathrm{M}=1.42, \mathrm{t}(94)=3.315, \mathrm{p}<0.001)$.

There were no statistically significant differences between subjects with more (daily to $<=1 /$ month, $n=40$ ) or less frequent $(n=47)$ seizures in terms of HADS-Anxiety, HADSDepression, HADS-Total, or in any HADS item.

Nevertheless, according to MHI-5, individuals with more frequent seizures $(\mathrm{M}=18.03)$ had worse mental health $(\mathrm{M}=21.32, \mathrm{t}(85)=2.684, \mathrm{p}=0.009)$.

There were no statistically significant differences between those who had a lesional epileptic focus $(n=35)$ and those who did not ( $\mathrm{n}=49)$ on MHI-5 or any HADS item or score, except for item 14, in which those who had a lesional epileptic focus had a higher mean ( $\mathrm{M}=1.11$ vs. $\mathrm{M}=0.61$, 
$\mathrm{t}(82)=2.200, \mathrm{p}=0.03)$.

\section{DISCUSSION}

The present cross-sectional study investigated the usefulness of the HADS-Portuguese version as a screening instrument for Portuguese outpatients with focal epilepsy. To do so, the HADS was compared with another, briefer and widely used, screening instrument, the MHI-5, namely through the analysis of the relationships between HADS vs. MHI-5 indicators and several demographic and clinical variables.

The results indicate that the Portuguese HADS (and MHI-5) is understandable and well accepted by outpatients with clinical evidence of focal epilepsy and a wide age and schooling range, independent of their sex, marital and professional status, and disease characteristics. In fact, the sample is quite heterogeneous demographically and clinically. For instance, even though the sample, as a whole, can be considered a sample of individuals with clinical evidence of moderate severity focal epilepsy, it encompasses individuals with indicators of considerable severity (e.g., more than a seizure type, frequent seizures, polytherapy, toxic AED serum levels).

More specifically, the absence of statistically significant correlations between HADS and MHI-5 and schooling supports their use regardless of the respondents' formal education. However, it should be pointed out that, in the present study, the instruments were not self-administered.

Regarding the comparison between HADS and MHI-5, the first interesting finding concerns their internal consistency, which is the same for both scales and a good one (cf. Table 3). Additionally, participants of the study answered to both scales' items using the full range of response choices.

Even though the MHI-5 has a "positive" component (two of its items are from the Positive Well-being Scale of the MHI) and some HADS items are also "positive" (e.g., item 4), only the HADS offers the possibility of classifying respondents into severity classes, an important indicator in clinical and research settings (cf. Table 4). In this context, it is necessary to emphasize an important factor: there are Portuguese norms for the SF-36 version 1 ( $\left.{ }_{57}\right)$, which includes the MHI-5, and there are still no such norms for the Portuguese HADS

Concerning the relationship between HADS and MHI-5 items and scores, it is worth stressing that of all the 157 possible correlations, only 11 were not statistically significant (cf. Table 5). These exceptions should be analyzed. First, the non-significant correlation between item 11 of the HADS and item 3 of the MHI-5 is not in the expected direction. Second, item 11 of the HADS also does not present a statistically significant correlation with items 1 , 2 , and 5 of the MHI-5, i.e., it only correlates with item 4. Third, this is also the only HADS item that does not correlate with all HADS scores (namely, HADSDepression). Consequently, these results question the adequacy of item 11 .

Furthermore, item 1 of the MHI-5 does not correlate with 4 HADS items $(2,4,8$, and 11), item 3 of the MHI-5 does not correlate with items 2,11 , and 14 of the HADS, and item 5 of the MHI-5 does not correlate with items 10 and 11 of the HADS. Therefore, these results offer some evidence that the items of the two scales are not assessing (exactly) the same construct(s). In fact, as was stressed previously, MHI-5 and HADS are built with different construct aspects. Even so, it is worth noticing that the MHI-5 global score correlates more strongly with HADS-Total than with HADS-Anxiety or Depression, giving some support to the use of HADSTotal as a index of mental health/negative affect $\left({ }_{15},{ }_{41}\right)$.

Another relevant set of findings concerns the association patterns between HADS/MHI-5 and demographic and/or clinical variables. Again, these patterns reflect the fact that MHI-5 and HADS are built with different construct aspects. In fact, MHI-5 correlated with age, as did HADSDepression, but not HADS-Total nor HADS-Anxiety. One should also be aware of the insensitivity of MHI- 5 to disease onset and age at disease onset, while HADS-Depression and HADS-Total correlated with age at disease onset, an important finding in terms of mental health prevention. In addition, HADS-Depression was the only indicator sensitive to marital status.

Inversely, MHI-5 showed that those on monotherapy had better mental health than those on polytherapy, which makes perfect sense, especially when one considers that polytherapy is usually associated with more severe epilepsy. Strangely, no HADS item or score pointed in the same direction.

The results regarding the last seizure also question the consistency of the scores obtained. In fact, there were no statistically significant differences between those who had the last seizure less than a month before assessment and those who had it more than a month before assessment in HADS-Anxiety, but those with a more recent seizure 
reported worse mental health according to the MHI-5 and HADS-Total, and more depression. Another unforeseen discrepancy relates to seizure frequency: HADS scores and items were not sensitive to seizure frequency and MHI-5 was (in the expected direction).

Still, not all association patterns differed. Indeed, regarding sex differences, there was consistency between HADS and MHI-5 scores. Additionally, MHI-5 and HADS scores were both not sensitive to professional status, seizure types, and lesional epileptic focus status.

Even though all statistically significant associations (correlations or differences) between HADS items and demographic/clinical variables made clinical sense and/or were in the expected direction considering the scientific literature, the fact that there were incongruities between items' associations and scores' associations calls for reflection. Actually, one can hypothesize that the information lost when aggregating items, namely by ignoring some items ability to discriminate between certain groups of patients, may be clinically relevant. For instance, the type of anxiety symptoms a patient experiences (assessed by a self-report scale as the ones used) can be an important aid in planning the best possible intervention. Consequently, the HADS, with its 7+7 items, allows for the systematization and analysis of some clinically relevant aspects a briefer instrument, such as the MHI-5, does not.

In general, the present results showed that: (a) the internal consistency of the HADS was comparable to those reported in the literature $\left({ }_{38},{ }_{45}\right)$; b) its face validity seemed appropriate $\left({ }_{45}\right)$; and c) its construct validity (considering the MHI-5) acceptable. Nonetheless, the associations between HADS scores and some demographic and clinical variables questions its construct validity. In fact, it is important to note that the instrument didn't correlate with some demographic and clinical variables known to influence depression and anxiety.

This study aimed to explore the usefulness of the HADS not only as a screening instrument, but also as an intervention aid for Portuguese outpatients with focal epilepsy. In this context, the raw scores and the relationships found between HADS scores and the participants' demographic and clinical characteristics are worth analysing (the latter also as markers of the instrument construct validity). In fact, the raw scores suggest that anxiety and depression are common psychological disorders in focal epilepsy (cf. Table 4), which is in accordance with previous studies $\left({ }_{1}, 2,{ }_{8},{ }_{9},{ }_{20},{ }_{24},{ }_{27}\right.$,
$28,29,38)$. The results also showed there are several demographic and clinical characteristics associated with these disorders. In the sample studied, anxiety (more frequent) was associated with sex (women worse), replicating previous findings $(27,28,37,38)$.

Still, in the same sample, anxiety was not associated with: age, schooling, marital status, professional status, age at disease onset, disease onset $\left({ }_{28}\right)$, type of therapy, last seizure recency, seizure types, seizure frequency, or lesional epileptic focus status. These results differ from those of Mensah et al. ( ${ }_{28}$ ) (educational attainment, unemployment), Jacoby et al. ( ${ }_{26}$ ) (seizure frequency, disease onset/epilepsy duration), Ridsdale et al. ( ${ }_{25}$ ) (age, having more recent seizures), Reuber et al. ( ${ }_{30}$ ) (underlying epileptogenic lesion, AED therapy), and Kimiskidis et al. ( $\left.{ }_{27}\right)$ (seizure frequency, type of therapy/polypharmacy).

Depression, on the other hand, was associated with: age (older worse), sex (women worse), marital status (married worse than single), age at disease onset (older worse), and last seizure recency (more recent worse). These results agree with those from Jacoby et al. ( $\left.{ }_{26}\right)$ as far as age and age at seizure onset are concerned. Similarly, Kimiskidis et al. ( $\left.{ }_{27}\right)$ found that female gender showed a trend towards being significantly associated with depression. Still, Mensah et al. ${ }_{29}$ ) found that depression was not associated with sex or marital status, but it was associated with having had a recent seizure.

Inversely, in the present sample, depression was not associated with: schooling, professional status, disease onset, type of therapy, seizure types, seizure frequency, or lesional epileptic focus status. These results are not in accordance with those of Jacoby et al. ( ${ }_{26}$ ) (seizure frequency, disease onset/epilepsy duration), or Kimiskidis et al. ( $\left.{ }_{27}\right)$ (seizure frequency, high number of AED/type of therapy). Similarly, Ridsdale et al. ${ }_{25}$ ) found that being younger, woman, and having more recent seizures is not significantly associated with higher scores of depression. Mensah et al. ${ }_{29}$ ) found that depression was not associated with monotherapy or polytherapy, but it was associated with unemployment. Reuber et al. ( ${ }_{30}$ ) found that depression related to underlying epileptogenic lesion, and AED therapy.

Finally, negative affect (HADS-Total) was associated with: sex (women worse), age at disease onset (older worse), and last seizure recency (more recent worse). Nevertheless, in the same sample, negative affect was not associated with: 
age, schooling, marital status, professional status, disease onset, type of therapy, seizure types, seizure frequency, or lesional epileptic focus status.

It should be noted that no HADS item or score showed statistically significant differences between those on monotherapy and those on polytherapy (generally, a sign of more severe epilepsy). The fact that 28 out of the 38 individuals on polytherapy were on 2 AED does not seem sufficient to understand this unexpected result. The reasons for this and other inconsistencies between the present results and some of the published literature should be explored in future studies.

For now, one can assume the differences in the sample demographics and/or clinical characteristics and in methodology account for some of the differences between the studies. Be as it may, these contradictions should alert clinicians not to automatically generalize research findings to their patients, stressing the importance of assessing each patient's affective status.

To optimize the use of the Portuguese HADS with focal epilepsy patients, it would be important to be familiar with the response patterns of Portuguese individuals, especially with focal epilepsy, to the HADS considering the demographic variables included in the present study $\left({ }_{25},{ }_{26}\right.$, ${ }_{40}$ ). Additionally, it would be important to know if HADS scores vary during the natural course of (focal) epilepsy ${ }_{21}$, $\left.{ }_{22},{ }_{23}, 25,26\right)$. It is also of the upmost importance to clarify the processes underlying the anxiety and depression scores reported by different epilepsy samples $\left({ }_{1},{ }_{2},{ }_{3}, 7,{ }_{11},{ }_{12},{ }_{14}\right)$ and to identify the prevalence of the different types of anxiety and/or depression (pre, post, inter, ictal, with no relation to seizures), since they differ regarding the underlying pathophysiological mechanisms $\left({ }_{1},{ }_{2},{ }_{3},{ }_{7},{ }_{14}, 18\right.$ $, 27)$.

In conclusion, even though the present results suggest that the HADS should be more thoroughly studied, they support the use of the Portuguese HADS as a screening tool to be used with focal epilepsy patients. It seems that its routine use could help optimize health care and control for some biases in research, without consuming much time, a key factor in health care. Considering that the HADS should not be used as a diagnostic tool, its substitution for the MHI-5 as a screening tool of mental health could be considered acceptable, especially in situations in which time constraints are crucial. Nevertheless, this substitution leads to a considerable loss of information and specificity that has to be carefully weighted. The time frame of interest is another important variable, since the two instruments refer to different periods of time. Above all, the results underscore the importance of assessing patient affective status, since anxiety and depression are common in focal epilepsy outpatients and provide additional insight to the association of anxiety and depression with certain demographic and clinical variables.

\section{ACKNOWLEDGMENTS}

The authors would like the acknowledge the financial support Financial support from the Science and Technology Foundation (Portugal)/Fundação para a Ciência e a Tecnologia to the $\mathrm{PhD}$ research project in the context of which the data presented in this article was obtained (Grant PRAXIS XXI / BD / 18536 / 98 - Sub-Programa Ciência e Tecnologia do $2^{\circ}$ Quadro Comunitário de Apoio).

\section{CORRESPONDENCE TO}

Prof. Doutora Rute F. Meneses Faculdade de Ciências Humanas e Sociais - Universidade Fernando Pessoa Praça 9 de Abril, 349 4249-004 Porto Portugal E-mail:

rmeneses@ufp.edu.pt

\section{References}

1. Hermann BP. Quality of life in epilepsy. J Epilepsy 1992;5:153-165.

2. Torta R, Keller R. Behavioral, psychotic, and anxiety disorders in epilepsy: Etiology, clinical features, and therapeutic implications. Epilepsia 1999;40(Suppl. 10):S2S20.

3. Perrine K, Kiolbasa T. Cognitive deficits in epilepsy and contribution to psychopathology. Neurology 1999;53(Suppl. 2):S39-S48.

4. Meneses RF, Ribeiro JP, Martins da Silva A. A importância da avaliação do humor negativo em indivíduos com epilepsia. In: Avanços recentes em psicologia clínica e da saúde (Coords. ML Marinho, RM Silva, SR Souza, GR Isquierdo, JSV Kanamota, FB Souza, PS Marinho; vol. 2). Londrina. Granada, Editora UEL / APICSA, 2001: p. 168. 5. Starratt C. Emotional disorders associated with neurological diseases. In: Clinical neuropsychology: A pocket handbook for assessment (Eds. PJ Snyder, PD Nussbaum). Washington, American Psychological Association, 1998: pp. 613-628.

6. Martins da Silva A, Taveira MC, Matos PM, Mendonça

$\mathrm{D}$, et al. Necessidades de intervenção e investigação psicossocial em epilepsia: Resultados de um inquérito em Portugal. Boletim da Epilepsia 1997;2(2):29-33.

7. Perrine KR. Psychopathology in epilepsy. Semin Neurol 1991;11(2):175-181.

8. Onuma T. Classification of psychiatric symptoms in patients with epilepsy. Epilepsia 2000;41(Suppl. 9):43-48.

9. Hermann BP, Seidenberg M, Bell B. Psychiatric comorbidity in chronic epilepsy: Identification, consequences, and treatment of major depression. Epilepsia 2000;41(Suppl. 2):S31-S41.

10. Alldredge BK. Seizure risk associated with psychotropic drugs: clinical and pharmacokinetic considerations. 
Neurology 1999;53(Suppl. 2):S68-S75.

11. Baker GA, Jacoby A. Epilepsy. In: Comprehensive clinical psychology: Health psychology (Eds. DW Johnston, M Johnston; vol. 8). Amsterdam, Elsevier, 1998: pp. 487-503.

12. Blumer D. Personality in epilepsy. Semin Neurol 1991;11(2):155-166.

13. Hermann BP, Whitman S. Behavioral and personality correlates of epilepsy: A review, methodological critique, and conceptual model. Psychol Bull 1984;95(3):451-497.

14. Robertson MM. Depression in patients with epilepsy: An overview. Semin Neurol 1991;11(2):182-189.

15. RF Meneses, Promoção da qualidade de vida de doentes crónicos: Contributos no contexto das Epilepsias Focais,

Universidade Fernando Pessoa \& Fundação para a Ciência e a Tecnologia, Porto, 2005.

16. Cramer JA. Quality of life assessment in clinical practice. Neurology 1999;53(Suppl. 2):S49-S52.

17. O Devinsky, Behavioral neurology: 100 maxims, Mosby-Year Book, St. Louis, 1992.

18. Kanner AM, Nieto JCR. Depressive disorders in epilepsy. Neurology 1999;53(Suppl. 2):S26-S32. 19. Wiegartz P, Seidenberg M, Woodard A, Gidal B, Hermann B. Co-morbid psychiatric disorder in chronic epilepsy: Recognition and etiology of depression. Neurology 1999;53(Suppl. 2):S3-S8.

20. Fu CW, Tan AW, Sheng F, Luan RS, Zhan SY, Chen WQ, et al. The prevalence of anxiety symptoms and depressive symptoms in patients with somatic disorders in urban China: a multi-center cross-sectional study. Int J Psychiatry Med 2007;37(2):185-199.

21. O'Donoghue MF, Goodridge DMG, Redhead K, Sander JWAS, Duncan JS. Assessing the psychosocial consequences of epilepsy: a community-based study. $\mathrm{Br} \mathrm{J}$ Gen Pract 1999;49:211-214.

22. Trueman P, Duthie T. Use of the Hospital Anxiety and Depression Scale (HADS) in a large, general opulation study of epilepsy. Quality of Life Newsletter 1998;19:9-10.

23. Blake RV, Wroe SJ, Breen EK, McCarthy RA. Accelerated forgetting in patients with epilepsy: Evidence for an impairment in memory consolidation. Brain 2000;123:472-483.

24. Cramer JA, Brandenburg N, Xu X. Differentiating anxiety and depression symptoms in patients with partial epilepsy. Epilepsy Behav 2005;6(4):563-569.

25. Newly diagnosed epilepsy: Can nurse specialists help? A randomized controlled trial. Epilepsia

2000;41(8):1014-1019.

26. Jacoby A, Baker GA, Steen N, Potts P, Chadwick DW.

The clinical course of epilepsy and its psychosocial correlates: Findings from a U.K. community study. Epilepsia 1996;37(2):148-161.

27. Kimiskidis VK, Triantafyllou NI, Kararizou E, Gatzonis S-S, Fountoulakis KN, Siatouni A, et al. Depression and anxiety in epilepsy: The association with demographic and seizure-related variables. Ann Gen Psychiatry 2007;6:28. 28. Mensah SA, Beavis JM, Thapar AK, Kerr MP. A community study of the presence of anxiety disorder in people with epilepsy. Epilepsy Behav 2007;11(1):118-124. 29. Mensah SA, Beavis JM, Thapar AK, Kerr M. The presence and clinical implications of depression in a community population of adults with epilepsy. Epilepsy Behav 2006;8(1):213-219.

30. Reuber M, Andersen B, Elger CE, Helmstaedter C. Depression and anxiety before and after temporal lobe epilepsy surgery. Seizure 2004;13(2):129-135.

31. Panelli RJ, Kilpatrick C, Moore SM, Matkovic Z, D'Souza WJ, O'Brien TJ. The Liverpool Adverse Events
Profile: relation to AED use and mood. Epilepsia

2007;48(3):456-463.

32. Mosaku KS, Fatoye FO, Komolafe M, Lawal M, Ola BA. Quality of life and associated factors among adults with epilepsy in Nigeria. Int J Psychiatry Med

2006;36(4):469-481.

33. Tracy JI, Dechant V, Sperling MR, Cho R, Glosser D.

The association of mood with quality of life ratings in epilepsy. Neurology 2007;68(14):1095-1096.

34. Meldolesi GN, Picardi A, Quarato PP, Grammaldo LG,

Esposito V, Mascia A, et al. Factors associated with generic and disease-specific quality of life in temporal lobe epilepsy. Epilepsy Res 2006;69(2):135-146.

35. Johnson EK, Jones JE, Seidenberg M, Hermann BP. The relative impact of anxiety, depression, and clinical seizure features on health-related quality of life in epilepsy.

Epilepsia 2004;45(5):544-550.

36. Zimmermann F, Endermann M. Self-proxy agreement and correlates of health-related quality of life in young adults with epilepsy and mild intellectual disabilities.

Epilepsy Behav 2008;13(1):202-211.

37. Keedwell P, Snaith RP. What do anxiety scales measure? Acta Psychiatr Scand 1996;93:177-180.

38. Botega NJ, Bio MR, Zomignani MA, Garcia C Jr, Pereira WAB. Transtornos do humor em enfermaria de clínica médica e validação de escala de medida (HAD) de ansiedade e depressão. Rev Saude Publica

1995;29(5):355-363.

39. A Bowling, Measuring disease: A review of diseasespecific quality of life measurement scales (2nd ed.), Open University, Buckingham, 2001.

40. Herrmann C. International experiences with the Hospital Anxiety and Depression Scale. A review of validation data and clinical results. J Psychosom Res 1997;42(1):17-41. 41. Johnston M, Pollard B, Hennessey P. Construct validation of the hospital anxiety and depression scale with clinical populations. J Psychosom Res 2000;48:579-584. 42. Snaith P. Anhedonia: exclusion from the pleasure dome. A useful marker of biological depression. Br Med J 1992;305:134.

43. Snaith RP. The Hospital Anxiety and Depression Scale. J Psychosom Res 2002;52(5):401.

44. Snaith RP, Protheroe DN. The Hospital Anxiety and Depression Scale. Hospital Update 1995, May:240-242. 45. RP Snaith, AS Zigmond, The Hospital Anxiety and Depression Scale with The Irritability-Depression-Anxiety Scale and The Leeds Situational Anxiety Scale Manual, NFER-NELSON, Berkshire, 1994.

46. Herrmann-Lingen C, Klemme H, Meyer T. Depressed mood, physician-rated prognosis, and comorbidity as independent predictors of 1-year mortality in consecutive medical inpatients. J Psychosom Res 2001;50:295-301. 47. Zigmond AS, Snaith RP. The Hospital Anxiety and Depression Scale. Acta Psychiatr Scand 1983;67:361-370. 48. White D, Leach C, Sims R, Atkinson M, Cottrell D. Validation of the Hospital Anxiety and Depression Scale for use with adolescents. Br J Psychiatry 1999;175:452-454. 49. Snaith $\mathrm{P}$. What do depression rating scales measure? Br J Psychiatry 1993;163:293-298.

50. Nitschke JB, Heller W, Imig JC, McDonald RP, Miller GA. Distinguishing dimensions of anxiety and depression. Cognit Ther Res 2001;25(1):1-22.

51. Bjelland I, Dahl AA, Haug TT, Neckelmann D. The validity of the Hospital Anxiety and Depression Scale: An updated review. J Psychiatr Res 2002;52:69-77.

52. Love AW, Kissane DW, Bloch S, Clarke D. Diagnostic efficiency of the Hospital Anxiety and Depression Scale in women with early stage breast cancer. Aust N Z J Psychiatry 
2002;6(2):246-250.

53. Pais-Ribeiro J, Silva I, Ferreira T, Martins A, Meneses $\mathrm{R}$, Baltar M. Validation study of a Portuguese version of the Hospital Anxiety and Depression Scale. Psychol Health Med 2007;12(2):225-237.

54. Williams C, Snaith P. Anxiety: one of medicine's blind spots. Br J Hosp Med 1995;53(4):126-127.

55. Ribeiro JLP. Mental Health Inventory: Um estudo de adaptação à População Portuguesa. Psicologia, Saúde \& Doenças 2001;2(1):77-99.

56. JE Jr. Ware (with KK Snow, M Kosinski, B Gandek), SF-36 Health Survey manual and interpretation guide, Author, Boston, 1993.

57. JLP Ribeiro, O importante é a saúde: Estudo de adaptação de uma técnica de avaliação do Estado de Saúde. SF-36, Fundação Merck Sharp \& Dohme, Lisboa, 2005. 


\section{Author Information}

Rute F. Meneses, PhD

FCHS-Universidade Fernando Pessoa

José Pais-Ribeiro, Ph.D.

FPCE-Universidade do Porto

\section{A. Martins da Silva, MD, PhD}

Clinical Director of Hospital Geral de Santo António, ICBAS-Universidade do Porto

Anna R. Giovagnoli, MD, PhD

Division of Neuropathology \& Neurology, National Neurological Institute "Carlo Besta" 